OOMPUTATIONAL MATHEMATICS

BANACH OENTICR PUBLICATIONS, VOLUME 13

PWN-POLISH SOIENTIFIO PUBLISHIRS

WARSAW 1884

\title{
ПАКЕТЫ ПРИКЛАДНЫХ ПРОГРАММ LTРВVР И SPARSE
}

А. А. АБРА МОВ, Н. Г. БУРАГО, В. В. ДИТКИН, А. Л. ДЫШКО, A. IO. ЕРЕ МИН, А. Ф. ЗАБОЛОЦКАЯ, Н. Б. КОНЮХОВА, Н. Я. МАР БЯШ КИ Н, Б. С. ПАРИИСКИИ, В. И. УЛЬЯНОВА, И. И. ЧЕЧЕЛЬ

Вынислительньй Центр АН СССР, ул. Вавилова 40, Москва, СССР

\section{Введение}

В Вычислительном центре АН СССР создан пакет прикладных программ Линейные обыкновенные краевые задачи, предназначенный для решения двухточечных краевых задач для линейных обыкновенных дифференциальных и разностньх уравнений, а также систем таких уравнений. Пакет является частью системы пакетов прикладных программ для реализации общих методов вычислительной математики и включен в вычислительный номплекс САФРА (см. [1]). Пакет состоит ив двух частей: систематиэированной библиотеки процедур и Iтрограмм с именем LTPBVP (Linear Two Point Boundary Value Problem) и пакета SPARSE. Программы пакета LTPBVP написаны на языке ФОРТРАН и АЛГОЛ. Пакет SPARSE - на нзыке ФОРТРАН. Реализация панета предусмотрена на ЭВМ БЭСМ-6.

В пакет LTPBVP включены как методы для решения краевых дифференциальных задач, т.е. задач, сформулированных в виде системы линейных обыкновенных дифференциалыных уравнений с коэффициентами, заданными каними-либо формулами, и соответствующих граничных условий, так и для решения разностных краевых задач, сформулированных в виде системы линейных алгебраических уравнений специального типа. В пакете LTPBVP реализованы методы для решения двухточечных краевых задач, задач с условием периодичности и общей двухточечной задачи с нераспадающимися краевыми условиями. Пакет содержит в виде отдельных процедур и подпрограмм почти все известные авторам варианты метода прогонки. Процедуры пакета LTPBVP сданы в Государственный фонд алгоритмов 
и программ при ВЦ АН СССР (тексты процедур, комментарии к этим тенстам и тестовые примеры). В [25]-[40] приведены краткие сведения об этих материалах.

Панет SPARSE предназначен для решения спстем линейных алгебраических уравнений с разреженными матрицами. Такие задачи возникалот, например, при разностной аппроксимации дифференциальных граевых задач. Способы представления данных, структура пакета SPARSE и методы решения описаны в [22], [23].

Процедуры и подпрограммы пакетов LTPBVP и SPARSE использовались для решени разнообразных тестовых задач. В настоящее время пакеты успешно используются для решения различных прикладных задач.

\section{1. Пакет LTPBVP}

1.1. Реалнзованные методы. Методы, использованные в пакете LTPBVP, можно условно разделить на две группы:

(a) глассические прогонги, включая глассическую диффференциальнуг прогонку, потоновуг прогонку и классическую прогонку с условием периодичности, а также их разностные аналоги;

(б) ортогональные прогонки.

Для решения одного уравнения второго порядка (самосопряженная граевая задача)

$$
\begin{gathered}
\left(p(x) y^{\prime}\right)^{\prime}-q(x) y=f(x), \quad a \leqslant x \leqslant b, \\
\alpha_{1} y(a)+\beta_{1} y^{\prime}(a)=\gamma_{1}, \quad \alpha_{2} y(b)+\beta_{2} y^{\prime}(b)=\gamma_{2},
\end{gathered}
$$

где $x$ - вещественная переменная, $p(x), q(x), f(x)$ - заданные на $[a, b]$ вецественные функции, $\alpha_{i}, \beta_{i}, \gamma_{i}$ - вещественные числа, $a_{i}^{2}+\beta_{i}^{2} \neq 0$, предусмотрены следующие варианты метода прогонки:

- жлассический вариант метода прогонки (см. [2]-[5], [25]);

- потоковый вариант метода прогонки для случая, когда $p(x)=$ $=1 / \sigma(x)$ и $\sigma(x) \geqslant 0$ (см. [6], [26]);

- циклическая прогонка для случая вадачи с граничными условиями периодичности (см. [7], [25]).

Для решения общей двухточечной задачи с распадагщимися грапичными условиями для одного уравнения второго порядка

$$
\begin{gathered}
y^{\prime \prime}+p(x) y^{\prime}+q(x) y=f(x), \quad a \leqslant x \leqslant b, \\
a_{1} y(a)+\beta_{1} y^{\prime}(a)=\gamma_{1}, \quad a_{2} y(b)+\beta_{2} y^{\prime}(b)=\gamma_{2},
\end{gathered}
$$

где $x$-вещественная переменная, $p(x), q(x), f(x)$ - непрерывные на $[a, b]$ функции, $a_{i}, \beta_{i}, \gamma_{i}-$ числа, $\left|a_{i}\right|^{2}+\left|\beta_{i}\right|^{2}=1$, применяются два 
алгоритма ортогональной прогонки: вариант ортогонального переноса граничных условий для задачи с вещественными коэффициентами (см. [8], [27]) и его модификация для задачи с комплексньми коэффициентами (см. [9], [27]).

Для решения краевых задач для систем второго порядка вида

$$
\begin{gathered}
y^{\prime \prime}-P(x) y=f(x), \quad a \leqslant \infty \leqslant b, \\
A_{1} y(a)+B_{1} y^{\prime}(a)=C_{1}, \quad A_{2} y(b)+B_{2} y^{\prime}(b)=C_{2},
\end{gathered}
$$

где $x$-вещественная переменная, $P(x)$-заданная на $[a, b]$ функция - квадратная матрица порядка $n, A_{i}, B_{i}$ - квадратные матрицы порядка $n, f(x)$ - заданная на $[a, b]$ функция-столбец высоты $n, C_{1}, C_{2}$ - столбцы высоты $n$, испольәуіотся следующие методы:

- матричная классическая дифференциальная прогонка для задач с действительными коэффициентами при $B_{1}=B_{2}=E$ (см. [13], [10], [28]);

- два метода ортогональной прогонки для самосопряженной задачи: $P=P^{*}, A_{1} B_{1}^{*}=B_{1} A_{1}^{*}, A_{2} B_{2}^{*}=B_{2} A_{2}^{*}$ (см. [11], [29] для задачи с действительньми коэффициентами и [12], [30] для задачи с комплексньми коэфффициентами).

Для решения вещественных краевых задач для систем первого порядка вида

$$
\begin{gathered}
y^{\prime}-P(x) y=f(x), \quad a \leqslant x \leqslant b, \\
\psi_{a} y(a)=g_{a}, \quad \psi_{b} y(b)=g_{b},
\end{gathered}
$$

где $P(x)$ - непрерывная на $[a, b]$ квадратная матрица порядка $n$, $f(x)$ - непрерывный на $[a, b]$ столбец высоты $n, \psi_{a}$ - матрица размера $k \times n, \psi_{b}$ - матрица размера $(n-k) \times n, g_{a}, g_{b}$ - столбцы высоты соответственно $k$ и $n-7$, примепяются следующие методы:

- нлассический вариант метода прогонки (см. [13], [14], [31]);

- три различных метода ортогональной прогонкт (см. [15], [5], $[16],[32],[33],[34])$;

- дифференциальная прогонка с промежуточной ортогонализацией иг нормировкой базисных решений (см. [17], [35]).

Для решсния двухточечных краевых задач для систем линейных обынговенных дифференциальных уравнений с нераспадагшимися граничными условиями, т.е. задач вида

$$
\begin{gathered}
y^{\prime}-P(x) y=f(x), \quad a \leqslant x \leqslant b, \\
A y(a)+B y(b)=C,
\end{gathered}
$$

где $P(x), A, B$ - квадратные матрицы порядка $n, f(x), C$ - столбцы высоты $\eta$, используется хіорошо известный прием, который сводит әту 
задачу ж задаче вдвое большей размерности с распавшимися граниұными условиями (см. [18], [36]). Прием состоит в следугшем. Введем

$$
\begin{gathered}
y_{1}=\left[\begin{array}{c}
y(x) \\
y(a+b-x)
\end{array}\right], \quad P_{1}(x)=\left[\begin{array}{c:c}
P(x) & 0 \\
\hdashline 0 & -P(a+b-x)
\end{array}\right], \\
f_{1}=\left[\begin{array}{c}
f(x) \\
-f(a+b-x)
\end{array}\right], \quad Q=\left[\begin{array}{lll}
-D &
\end{array}\right], \quad R=\left[\begin{array}{ll}
A & B
\end{array}\right] .
\end{gathered}
$$

Тогда получим задату

$$
\begin{gathered}
y_{1}^{\prime}-P_{1}(x) y_{1}=f_{1}, \quad a \leqslant x \leqslant(a+b) / 2, \\
R y_{1}(a)=C, \quad Q y_{1}((a+b) / 2)=0 .
\end{gathered}
$$

Для решения әтой задачи в пакете используется метод ортогонального переноса граничных условий.

Во всех подпрограммах, каж правило, для решения задач Коши применяется метод Рунге-Кутта с автоматическим выбором шага.

Для решения разностных граевых задач применяотся следудщие разностные прогонки:

- трехточечная скаллрная прогонка (см. [2], [3], [19], [37]);

- трехточеqная потоковая прогонна (см. [20], [38]);

- трехточечная скалярная прогонка с условием периодичности (cM. [7], [39]);

- многоточечная скалярная прогонка (см. [21], [40]);

- трехточедная матричная прогонка (см. [2], [19], [37]).

Для решения возникагоих вспомогательных линейных алгебраических уравнений применяется метод Гаусса с выбором главного элемента.

1.2. О работе процедур. При решении конкретных вадач возникает вопрос, каким именно методом целесообразно воспользоваться. Для отдельных типов задач можно отдать предпочтение одним методам перед другими. Однако часто заранее трудно что-либо сказать о нужных свойствах задач. Хорошо обусловленная задача не всегда мояет быть решена классическими методамп прогонги. С „гарантией" эти методы приведут н положительному результату лишь при выполнении достаточных условий, налагаемых на решаемуго задачу. Хорошо известно, например, что таким достаточным условием для плассической прогонй в случае одного уравнения второго порядка являлотся условия $p(x) \geqslant$ $\geqslant p_{0}>0, q(x) \geqslant q_{0}>0$ и неотрицательность чисел $-a_{1}, \beta_{1}, a_{2}$ и $\beta_{2}$. Поэтому в ряде процедур, которые реализугт „необщие" методы прогонки, предусматривается выход на метку, ноторая показывает, что 
данный метод не подходит для решения задачи. В этом случае рекомендуется применять какой-либо вариант ортогональной прогонки. C другой стороны, решаемая задача может ожазаться плохо обусловленной. Поэтому во всех подпрограммах пакета предусматрпвается выход на соответствуюшую метку, если решаемая задача окавывается с заданной точностьо плохо обусловленной.

Поясним сказанное на примере. Поскольку классический вариант метода прогонки хорошо иэвестен, то остановимся на решении этим методом одного уравнения второго порядка. Все остальные процедуры и подпрограммы пакета построены по тому же приндипу.

Обращение к процедуре имеет вид:

$$
\operatorname{clod}(a, b, m, m 1, u a, u b, y, y 1, p q f, \operatorname{eps}, \operatorname{lm}, \mathrm{lp}) \text {. }
$$

Имя процедуры - clod - использует буквы слов:

classical (классическая прогонка),

one (для одного уравнения),

differential (дифференциальный вариант).

Здесь:

$\boldsymbol{a}, \boldsymbol{b}$ - нонцы интервала интегрирования, не обязательно $\boldsymbol{a}<\boldsymbol{b}$;

$m$ - целое число, на $m$ частей разбивается интервал $[a, b]$, вначения $y(x)$ и $y^{\prime}(x)$ вычисляются в $m+1$ точках $x_{i}=a+i \times \frac{(b-a)}{m}$, $i=0,1, \ldots, m$;

$m 1=2^{k}(0 \leqslant k$ - целое число) - целочисленный параметр, который задает начальный шаг интегрирования $h=(b-a) /(m \times m 1)$ для решения задачи Коши (значения $m$ и $m 1$ не влияют на конечный результат, но удачный выбор этих величин может существенно сонратить время счета решаемой задаपи);

$u a[1: 3], u b[1: 3]$ - массивы, вадагощие граничные условия; $u a[1]=a_{1}, u a[2]=\beta_{1}, \quad u a[3]=\gamma_{1}, \quad u b[1]=a_{2}, \quad u b[2]=\beta_{2}$, $u b[3]=\gamma_{2}$

$y[0: m], y 1[0: m]$ - массивы значений $y(x)$ и $y^{\prime}(x)$ в точках $x_{i}$; pqf - процедура, определягшая коэффициенты уравнения;

eps - заданная абсолютная точность вычислений для $y(x)$ и $y^{\prime}(x)$, интегрирование задач Коши на один шаг производится с точностью

$$
\operatorname{eps} 1=\frac{\mathrm{eps}}{m \times m 1}=\frac{\mathrm{eps}}{(b-a) / h}
$$

lm - метка, на которуго передается управление в случае, когда данный метод не подходит для решения исходной задачи, а именно, когда $(b-a) / h>10^{10}$, где $h$ - значение шага интегрирования, полученное $k$ моменту выхода на метку $\mathrm{lm}$;

lp - метка, на которуго передается управление в случае, ногда 
исходная задача плохо обусловлена, а именно, когда определитель алгебраической системы второго порядка, возникагщий после прямой прогонки, меньше ерs.

І Ћак уже было отмечено, все процедуры и подпрограммы пакета устроены сходнып образом. При этом для удобства использования порядок перечисления формальных параметров в каждой процедуре и их смысл одннанов во всех процедурах.

\section{2. Паке' SPARSE}

Пагет SPARSE предназначен для решения систем линейных алгебраическх уравненй с разреженными матрицами на ӘВМ БЭСМ-6 в системе Фортран-Дубна (см. [41]).

B пакете SPARSE разреженность матриц учитывается специальными способами представлсния данных, исключающих хранение нулевых элементов матриц. В качестве способа решения используется модификация метода Гаусса (см. [23]). Все операцип пропзводятся только с ненулевыми элементами исходных, промеяуутчыы и конечных матриц. Әффективным средством оптимизации вычислений служит также предварительная обработка структуры матриц за счет перестановки ес строк и столбцов (см. [42]). Совонупность этих мер позволяет существенно уменьшить время счета и требуемую оперативнуго память əВM.

Для уменьшения влияния погрешностей вычислсний прсдусмотрены следугщие дополнительные возможности:

1. масштабирование строк и столбцов матрицы и правых частей системы,

2. масштабирование ведущих әлементов исключения,

3. различные варианты исклочения строк и выбора вепущего әлемента.

Пакет SPARSE включает в себя внутренніо систему отладни, с помощыо которой обрабатываготся все программные прерывания, возникающие при выполнении счетных модулей. В пакете имеются программы диагностики для установления мсста и причины прерывания. Пользователь может задавать такой режим работы пакета, прл котором после прерывания вычисления могут быть продоляены с нуяного места. Для этих целей специальные программы гонтролиругт работу слетных модулей и, изменяя управляющие параметры, возобновлялот работу пакета. Эти подпрограммы могут понолняться пользователями с учетом специфики решаемых задач.

В пакете пспользуется динамическое распределепие памяти и се аащита. Пакет монет быть легко вклочен в готовые программы без существенных изменений. 
Пакет SPARSE можно приспосабливать те решенио вадач с ааданной структурой матрицы. При этом әфффективность решения существенно возрастает.

Пакет SPARSE можно использовать для решения серии систем с одинаковой струнтурой матриц и различными правыми частями.

На базе пакета SPARSE в настоящее время разработан пакет SOLVER (см. [24]) для решения систем нелинейных функциональньх и обыкновенных дифференциальных уравнений с разреженньпи якобиевьпи матрицами.

Приведем два примера использования пакета SPARSE. Hиже в таблице приведены результаты решения системы 66 нелинейных функциональных уравнениц $F(x)=0$ (см. [43]) методом Ныотона.

\begin{tabular}{|c|c|c|}
\hline Номер итерации & $\|F(x)\|^{2}$ & $\begin{array}{c}\text { Времн, аатрапиваемое } \\
\text { на итерации (в сөн.) }\end{array}$ \\
\hline 1 & $2.98 \times 10^{+7}$ & 59 \\
2 & $2.92 \times 10^{+4}$ & 57 \\
3 & 2.63 & 5 \\
4 & $5.15 \times 10^{-2}$ & 4 \\
5 & $2.46 \times 10^{-5}$ & 5 \\
6 & $4.73 \times 10^{-10}$ & 4 \\
\hline
\end{tabular}

Из этих данных видно, что времена счета первой и последней итерации различалотся более чем на порядок, т.к. на последних итерациях в пакете SPARSE используются ранее полученные протоголы исключения.

Приведем результаты применения пакста SPARSE If расчетам кручения стержня прямоугольного сечения методом конечных элементов (см. [44]). Порядон системы - 674, число ненулевых әлементов в матрице - 4484, полуширина ленты исходной используемой матрицы -253 , профиль исходной матрицы - 13795, полуширина ленты после перестановки строк и столбцов (эта перестановка производится самим пакетом) -30 , профиль конечной матрицы - 9318. Время работы программы перестановки строг и стольцов - 35 секунд, время работы остальной части пакета SPARSE (решенне системы линейных алгебраических уравнений -37 секунд.

\section{Лнтература}

[1] М. М. Горбунов-Посадов, В. Я. Карпов, Д. А. Корягин, В. В. Красотченн о, В. В. Мартын ток, Пакет прикладных програмен $C A \Phi P A$. Системное наполнение, Препринт Ин-та прпнл. матем. АН СССР, 1977, № 85. 
[2] И. М. Гельфанд, О. В. Локуциевскии, Метод „провонки” для решелиля раяностных ураөнений; В нниге: С. К. Годунов, В. С. Р я бень ки й, Вөедения - теорио равностных схем, Фивматгиа, Москва 1962, Дополнөние 2, стр. 283-309.

[3] И. С. Береаин, Н. П. Жи дков, Методы вииислений, т. 2, Физматгиз, Москва 1960 , cтp. $387-3 \theta 0$.

[4] И. Бабушка, Э. Витасек, М. Прагер, Численные прочессы решетил дифференциальных уравненцй, „Мир”, Москва 1969, стр. 121-152.

[5] Н. С. Бахвалов, Численные нетоды, „Наука", Москва 1973, стр. 548-565.

[6] Л. М. Дегтярөв, А. П. Фаворски й, Потокооий вариант метода прогонки, ЖF. вычисл. мат. и мат. Фиа. 8.3 (1968), 870-684.

[7] А. А. Абрамов, В. Б. Ап дреев, $O$ nриянtиelluи метода прогонки к нахозкдению периодических решений дифференчиальных и ральостних уравнений, ibid. 3.2 (1963), 376-381.

[8] А. А. Абрамов, Вариант нетода прогоики, ibid. 1.2 (1061), 349-351.

[9] Е. С. Биргер, Н. Б. Новюхова, Численный расиет распространения ра. диоволн в веріпикально-неоднородной тропосфере, Радмотехника и олектроника XIV. 7 (1960), 1147-1156.

[10] Э. Полак, Численные методы оптимивачии, „Мир”, Москва 1974.

[11] J. H. Barrett, A Prüfer transformation for matrix differential equations, Proc' Amer. Math. Soc. 8 (1957), 510-518.

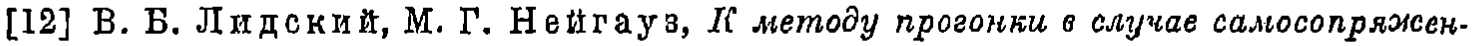
ной систельъ второго порлдка, 3К. вычисл. мат. и мат. (1из. 2.1 (1962), 161-165.

[13] J. Taufer, Lösung der Randwertprobleme für Systeme von linearen Differen. tialgleiohungen, Academia, Praha 1973.

[14] Н. В. В алишвили, Методы расиета оболочек оращенил иа ЭЦВ $M$, „Машипостроение", Москва 1976.

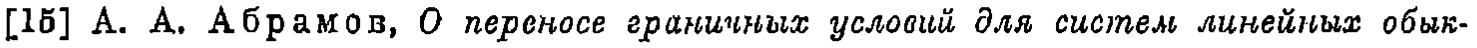
новенных дифференциальных уравнений, Ж. вычисл. мат. и мат. физ. 1.3 (1961), $542-545$.

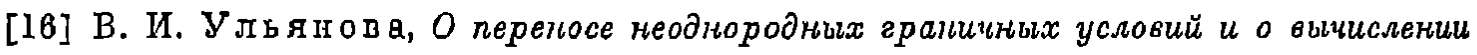
собстөенных функиий, ibid. 16.4(1876), 1057-1059.

[17] С. К. Годунов, О численьноме решении краевьх задаи для систем линейиых обыкновенкых дифференциальных ураөнений, УМН, XVI, 3 (99) (1961), 171-174.

[18] K. Moszyniski, A method of solving the boundary value problem for a system of linear ordinary differential equations, Algorytmy II. 3(1964), $25-43$.

[19] В. В. Огнева, Метод „прогонки” длл решенил разностных ураонений, Ж. вычисл. мат. Іи мат. физ. 4.4 (1967), 803-812.

[20] Л. М. Дегтярев, А. П. Фаворсий, Потоковий вариант нетода прогонки

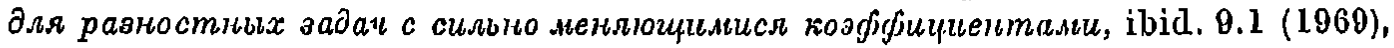
$211-218$.

[21] Н. Д. Сафонов, О методе прогонки длл решелия разностиьих краевых аядал, ibid. 4.2 (1964), 256-266.

[22] А. 1О. Еремин, Н. Я. М арьяшиин, Пакет програля SPARSE длю решенил

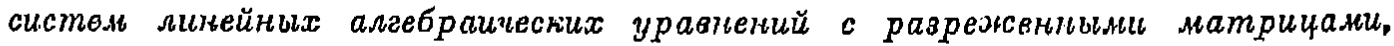
ВЦ AH GGGP, Москва 1978.

[23] -, -, Пакетпал обработка систем линейиьи алгебраических уравпений с рарреясенныции матричами, ЖН. вычисл. мат. п мат. физ. 18.6 (1978), 15611570 . 
[24] А. IO. Е ремин, Н. Я. Марьяшкин, Пакет програнль SOLVER системьь нелинейных фуукциональных и обыкновенных дифференчиальных уравнений с разреәсенными якобиевыљи матрицами, ВЦ АН СССР, Москва 1980.

[25] Е. В. Левшенко, В. И. Ульннова, Алгоритмы решенил линөйных само. сопрлэкенных краевых задаи и задач с условиями периодичности для обыкно. оенных дифференчиальных уравнений оторого порлдка методами классической оифберенцальной прогонки, Информ. бюлл. "Алгоритмы и программн” ВНТИЦентра, 1979, № 2, П003413.

[26] А. Ф. Заболоцная, Алворитм решения саносопрләсенньх дөухточечных линейных краевых вадаи для одного Әиффференчиального ураөнения второго порлдка (потоковал прогонка), Информ. бюлл. „Алгоритмы и программы” В НТИЦентра, 1979, № 2, ПО03475.

[27] Н. В. If он гохова, В. И. Ульянова, Алгоритмы рөшения линейиьгх краевых задаи для обыкновеиных дифференчиальных уравнений второго порлдка с өечестоенными и комплексными коэфффичиентами методами ортогональпой дифференчиальной прогонки, Информ. бюлл. „Алгоритмы и программы” ВНТИЦентра, 1979, № 2, ПО03409.

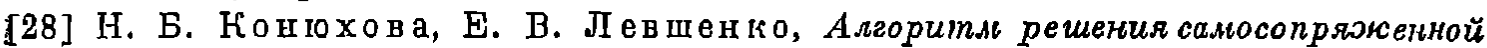
двухточеиной краевой вадачи для системь линейньх обыкновснных диффференчиальных уравнений второго порлдка с вечестөенныции коэфбфичиентани (латричнал классическал дифберенчиальнал прогонка), Информ. бюлл. „Алгорптмы и программы" ВНТИЦентра, 1979, № 2, ПОО3442.

[29] А. Ф. Заболоцкан, Алеоритм решения самосопрлюленной дөухточечной линейной краевой аадачи для систем линейных обикновенньх дифференчиальньгх ураоиений второво порлдка с вещественными ковффициентами, Информ. бюлл. "Алгоритмы и программы" ВНГИЦентра, 1979, $\mathrm{N}_{9}$ 5, П003853.

[30] А. Л. Дып по, Алгоритм нетода прогонки в случае самосопрязсенной системы

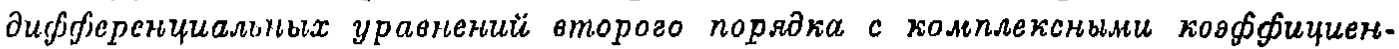
тами, Инцорм. бюлл. "Алгоритмы и программн” ВНТИЦентра, 1979, № 2, $\Pi 003474$.

[31] В. Н. Воробьев, Алгоритл решенил доухтоиечиыхх краеөых вадаи методом классической провонки для систен линейных обыкновенных дифференчиальньгх уравнений перөоео порлдка (GLSID), Информ. бюлл. „Алгоритмы и программы” ВНТИЦентра, 1978, № 5, ПОО3125.

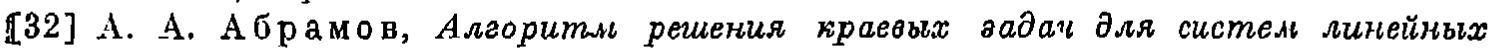
обыкновенных дифбјеренциальиых уравнений методом ортогонального переноса әраничныл условий, Информ. болл. „Алгоритмы п программы” ВНТИЦөнтра, 1970, № 2, ПОО3412.

[33] Б. С. П ар ии й ки й, Алгоритын реиселил краевых аадач длл систен линейных обыкновенных дифференчиальных уравнений методом дифференциальной орто. гональной прогонки, Информ. бглл. „Алгоритмы и программы” В НТ'ИЦентра, 1979, 수 2, П003492.

[34] А. А. А брамов, В. И. Ульянова, Алгоритм решения краеөых вадач для систем линейниг обыкновенных дифференчиальных уравнений модифичированиыль методоль ортогонального переноса гранииных условий, Ипформ. бюлл. "Алгоритмы и программы" ВНТИЦентра, 1979, 으 2, П003478.

[35] Н. Г. Бураго, В. М. Л гобимов, Алгоритл диябференчиальной прогонки с промежсуточной ортогоналиаачией и нормировкой базисных рөшений для систем обыкновенных линейных дифференциальных уравнений, Информ. бюлл. „Алгоритмы и программы" ВНТИЦентра, 1979, № 4, П003713. 
[36] Б. С. П ар и й с и й, Алгоритм реченил доухточечных краеөых вадаи длл систем линейнъгх дифوеренциальных уравнетий с нераспадалочимисл краевыми усло. өилми, Информ. бюлл. „Алгоритмы и программы” ВНТИЦентра, 1979, 수 2, П1003493.

[37] В. В. Дитнин, Алеоритмы решения равноспиных краевих задаи метоодами трехточечных прогонок, Информ. бюлл. "Алгоритмы и программы" ВНТИЦентра, 1979, № 3, ПО03554.

[38] А. Л. Дышко, Потоковый вариант метода провонки длл равностиыих аадаи с сильно менялочияисл ковфбичиентали, Информ. бюлл. „Алгоритмы пі программы" ВНТИЦештра, 1978, № 3, ПОО3638.

[39] И. И. Чечель, Алгоритм метода прогонки $к$ махољсденило периодических реиений разностных ураонений, Ин(јорм. бглл. „Алгоритмы и программы” ВНТИЦентра, 1979, № 2, П003490.

[40] -, Алгоритм метода прогонки для решения раяностних краевых аадаи, Информ. бюлл. „Алгоритмы и программн” ВНТИЦентра, 1979, 수 2, $\Pi 003480$.

[41] А. И. Салтыков, Г. И. Макаренко, Программирование на ләыке Фортран, "Наука", Моснва 1970.

[42] N. E. Gibbs, W. G. Polle, Jr., P. K. Stockmeyer, An algorithm for reducing the bandwidth and profile of a sparse matrix, SIAM J. Num. Anal, 13.2 (1976), 236-250.

[43] J. Erikse on, Solution of sparse systems of multilinear equations, Report Lith. Mat-R-1977-11, Linkoping Univer., Sweden.

[44] Л. Сегерлинд, Применение метода конеиньх өлементов, „Мир”, Москва 1979. 